\title{
Diagnostic ability of intraoperative ultrasound for identifying tumor residual in glioma surgery operation
}

\author{
Guangying Zhang ${ }^{1}$, Zhanzhan $\mathrm{Li}^{1}$, Daolin $\mathrm{Si}^{2}$ and Liangfang Shen ${ }^{1}$ \\ ${ }^{1}$ Department of Oncology, Xiangya Hospital, Central South University, Changsha, Hunan Province 410008, China \\ ${ }^{2}$ Department of Pediatric Neurology, Xiangya Hospital, Central South University, Changsha, Hunan Province 410008, China \\ Correspondence to: Liangfang Shen, email: Ifshen2008@163.com
}

Keywords: intraoperative ultrasound, glioma residual, diagnostic test, meta-analysis

Received: June 28, $2017 \quad$ Accepted: August 09, $2017 \quad$ Published: August 22, 2017

Copyright: Zhang et al. This is an open-access article distributed under the terms of the Creative Commons Attribution License 3.0 (CC BY 3.0), which permits unrestricted use, distribution, and reproduction in any medium, provided the original author and source are credited.

\section{ABSTRACT}

Achieving total glioma resection represents a major challenge to neurosurgeons with no distinct margin between tumor and surrounding brain tissue. Many imaging methods are employed in surgery visualization and resection control. We performed this meta-analysis to assess the diagnosis value of intraoperative ultrasound and judged whether ultrasound is a suitable tool in detecting glioma residual. The databases including PubMed, Embase, Web of Science, China National Knowledge Infrastructure (CNKI), Wanfang and Weipu were systematically searched to find out relevant studies and published up to May 5, 2017. A total of 14 studies involving 542 participants met the selection criteria and bivariate mixed effects models were used for analysis. The parameters and their corresponding $95 \%$ confidence interval (CI) were computed on Stata 12.0 software. The pooled sensitivity was 0.75 (95\%CI: $0.62-0.84)$, specificity was 0.88 (95\%CI: $0.79-0.94)$, positive likelihood ratios was 6.27 (95\%CI: 3.76-10.47), negative likelihood ratios was 0.29 (95\%CI: $0.20-0.42$ ), diagnostic odds ratios was 21.83 (95\%CI: 14.20-33.55) and area under the curve of summary receiver operator characteristic was 0.89 . Stratified meta-analysis showed sensitivity and area under the curve in low-grade glioma were both higher than high-grade glioma. The Deek's plot showed no significant publication bias $(t=-1.03$, $P=0.33$ ). Intraoperative ultrasound has high overall diagnostic value to identify glioma remnants, especially in low-grade glioma, which shows a benefit for prognosis and life quality of patients. In general, Intraoperative ultrasound is an effective tool for maximizing the extent of glioma resection.

\section{INTRODUCTION}

Glioma is the most common primary intracranial tumor account for about $40 \%$ of central nervous system tumors and $70 \%$ of malignant brain tumors [1], Whose treatment protocols are mostly based on surgical treatment, radiation therapy or temozolomide adjuvant chemotherapy [2]. However, the primary treatment of glioma is microscopic surgical resection, which must be maximally remove the tumor tissues and preserving normal nerve function. But it is a challenge to resect total tumor lesion due to the ambiguous boundary between tumor and normal tissue with glioma invasive growth [3-4]. Thus, the precise tumor localization and boundary identification are required to improve surgical strategies. The real-time images and position marks properties of intraoperative ultrasound (IOUS) were extensively used in tumor location, residue monitoring, guidance of aspiration biopsy and imaging blood flow of intracranial glioma [5-6], which is easy to operate and can be applied repeatedly. In comparison, intraoperative magnetic resonance imaging (iMRI) and navigational positioning system are limited by the expensive facility and need to avoid bias of brain transformation or shifting after skull opening in detecting intracranial glioma remnants[7-8]. In recent years, the clinical application of detection equipment for tumor residual may decrease the 
rate of malignant degeneration and prolong the median survival time and progression-free interval through the radical excision [9]. However, the diagnosis value of IOUS to detect the intracranial tumor removal is remains controversial due to the absence of cases and long-term follow-up data. And a point of ongoing discussion of iMRI, PET and navigational positioning system are superior to IOUS with insufficient data supporting. Thus, we performed this systematical review and meta-analysis to investigate the diagnostic values of IOUS in glioma operation.

\section{RESULTS}

\section{Selection and characteristics of studies}

A total of 542 articles were yielded in primary literature search, which included 147 records identified from PubMed, 36 records from Embase, 90 records from Web of Science, 106 records from China National Knowledge Infrastructure (CNKI), 88 records from Wanfang and 75 records from Weipu. After screening duplicate and unrelated topic, 37 articles were identified to further read by retrieving the full text. Finally, on the basis of above inclusion criteria, 14 articles were included with 1946 samples [10-14, 15-23]. The literature screening process and results are given in Figure 1.

The diagnostic accuracy of ultrasound for detecting glioma residual when the surgeons were considered excised completely should evaluate in all included studies. We know a patient could take more than one biopsy samples, only a few studies $(n=3)$ take patients as their object of study. When the patients and nidus on biopsy were both presented, we employed the latter, because the ultrasound results were one-to-one correspondence to every focus. We extracted the author, country, year of publication, mean age, sample size from the eligible articles, which were published between 1996 and 2016. The basic characteristics of the included studies are given in Table 1.

\section{Quality evaluation}

The summary of the quality assessment with regard to risk of bias and applicability concerns is shown in Supplementary Figure 1 and Supplementary Figure 2. We scored the questions as "low", "high", or "unclear" to examine included studies. As we can see, 1 study in patient selection is treated as high concern for applicability concerns. In summary, the quality of included studies met requirements for meta-analysis.

\section{Quantitative synthesis}

The latest Preferred Reporting Items for Systematic Reviews and Meta-Analyses Protocols were applied here. The study selection, data extraction, and risk of bias evaluation were performed by two authors independently to ensure the quality of this meta-analysis. Notable heterogeneity was observed on account of $\mathrm{I}^{2}=$ $89.21 \%, P<0.01$ for sensitivity and $\mathrm{I}^{2}=91.55 \%, P<$ 0.01 for specificity. Therefore, a random effects model was adopted for this meta-analysis. We calculated the pooled sensitivity of 0.75 (95\%CI: $0.62-0.84)$, pooled specificity of 0.88 (95\%CI: $0.79-0.94)$. The overall positive likelihood ratios (PLR) and negative likelihood ratios (NLR) were 6.27 (95\%CI: 3.76-10.47) and 0.29 (95\%CI: 0.20-0.42), respectively. The diagnostic odds ratios (DOR) was 21.83 (95\%CI: 14.20-33.55) (Figure 2 and Figure 3). Based on the sensitivity and specificity, we generated an summary receiver operator characteristic (SROC). The area under the curve (AUC) of the SROC was 0.89 (95\%CI: 0.85-0.91), which suggested that IOUS has a relatively high diagnostic value for detecting glioma residual (Figure 4). As showed on the Fagan plot, the prior probability was $20 \%$, posterior probabilities of PLR and NLR were $61 \%$ and $7 \%$, respectively (Figure 5). In general, IOUS demonstrated high diagnostic accuracy in detecting glioma residues during surgical operation.

\section{Stratified analysis}

In order to assess the impact of heterogeneity, we conducted subgroup analyses to further research the diagnostic power of IOUS in glioma residual resection. We suspected that study region, mean age, sample size and glioma grading could affect the pooled parameters of IOUS diagnosis evaluation. Further studies showed that parameters in mean age, sample size and region were no obvious difference between the pooled data, which suggesting those factors were not greatly impact on heterogeneity. However, the sensitivity of low-grade glioma reached a high accuracy (rose from 0.75 to 0.87 ). The meta-regression analysis revealed IOUS was more accurate for low-grade glioma than high-grade glioma to detect residual (AUC 0.93 vs. 0.83). But it limited to few samples, it will be more robust and credible with increased studies and standardized methodology. The detail parameters for each subgroup are summarized in Table 2 .

\section{Sensitivity analysis and publication bias}

We performed sensitivity analysis by excluding individual study sequentially. There was no remarkable variation for pooled parameters (the detail data were not given), indicating the pooled estimations were stable. The Deek's plot were used to evaluate the publication, as the Figure 6 indicates that was no significant publication bias exist $(\mathrm{t}=-1.03, P=0.33)$. 
Table 1: Characteristics of the included studies in the meta-analysis

\begin{tabular}{|c|c|c|c|c|c|c|c|c|c|c|c|}
\hline No. & Author & Year & Region & Mean age(y) & $\begin{array}{l}\text { Sample } \\
\text { size }\end{array}$ & Study design & $\begin{array}{l}\text { Study } \\
\text { population }\end{array}$ & TP & FP & $\mathbf{F N}$ & TN \\
\hline 1 & $\mathrm{He}$ & 2012 & China & $35.6 \pm 8.2$ & 38 & Prospective & Single center & 5 & 4 & 5 & 24 \\
\hline 2 & Qiu & 2015 & China & $45.1 \pm 13.1$ & 173 & Prospective & Single center & 20 & 10 & 13 & 130 \\
\hline 3 & Yang & 2014 & China & $43.47 \pm 13.83$ & 83 & Prospective & Single center & 13 & 10 & 8 & 52 \\
\hline 4 & Wang & 2009 & China & $39.0 \pm 10.9$ & 150 & Prospective & Single center & 48 & 7 & 21 & 74 \\
\hline 5 & Tian & 2009 & China & 41 & 189 & Prospective & Single center & 101 & 19 & 25 & 44 \\
\hline 6 & Guo & 2011 & China & 41.6 & 373 & Prospective & Single center & 48 & 5 & 36 & 284 \\
\hline 7 & Chen & 2007 & China & 39.6 & 216 & Prospective & Single center & 23 & 3 & 12 & 178 \\
\hline 8 & Liu & 2009 & China & $22-68$ & 80 & Prospective & Single center & 44 & 11 & 6 & 19 \\
\hline 9 & Woydt & 1996 & German & 45.8 & 78 & Prospective & Single center & 47 & 6 & 6 & 19 \\
\hline 10 & Becker & 1999 & German & 45.6 & 31 & Prospective & Single center & 24 & 2 & 1 & 4 \\
\hline 11 & Chacko & 2003 & India & $38.2 \pm 8.8$ & 96 & Prospective & Single center & 66 & 13 & 2 & 15 \\
\hline 12 & Venelin & 2011 & German & - & 11 & Prospective & Single center & 5 & 1 & 1 & 4 \\
\hline 13 & Shu & 2016 & China & $39.6 \pm 6.8$ & 360 & Prospective & Single center & 69 & 18 & 42 & 231 \\
\hline 14 & Jan & 2015 & German & 56 & 68 & Prospective & Single center & 12 & 1 & 37 & 18 \\
\hline
\end{tabular}

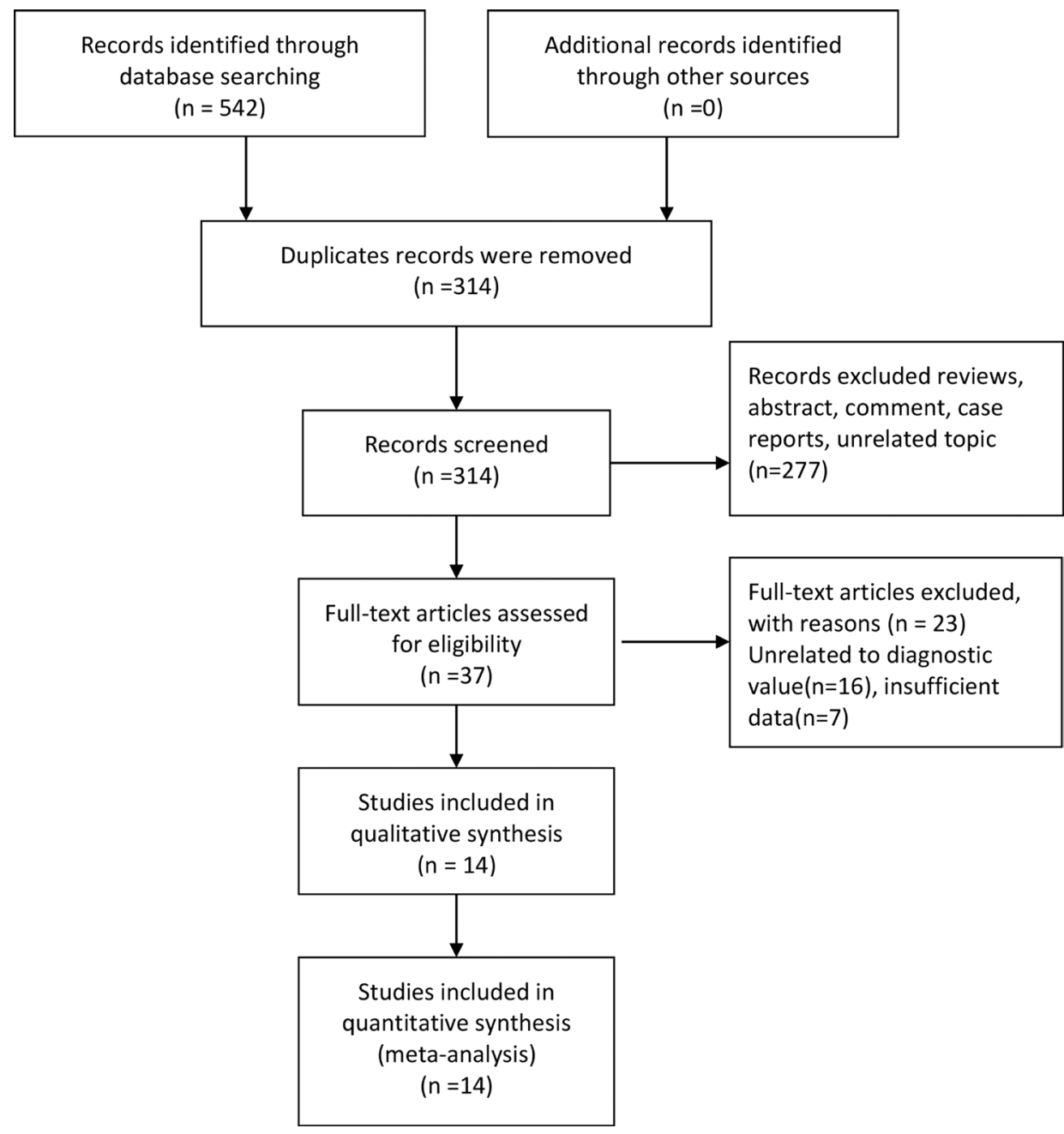

Figure 1: Flow diagram of studies selection process. 
Table 2: The pooled sensitivity, specificity, PLR, NLR and DOR, and their 95\% CI for each subgroup

\begin{tabular}{|c|c|c|c|c|c|c|}
\hline Category & SEN(95\% CI) & $\operatorname{SPE}(95 \% \mathrm{CI})$ & PLR(95\% CI) & $\operatorname{NLR}(95 \%$ CI) & $\operatorname{DOR}(95 \% \mathrm{CI})$ & $\operatorname{AUC}(95 \% \mathrm{CI})$ \\
\hline \multicolumn{7}{|l|}{ Region } \\
\hline Asian & $0.73[0.63-0.8]$ & $0.89[0.78-0.94]$ & $6.43[3.56-11.62]$ & $0.30[0.23-0.40]$ & $21.23[13.20-34.15]$ & $0.86[0.83-0.89]$ \\
\hline Europe & $0.66[0.58-0.74]$ & $0.82[0.69-0.91]$ & 3.59 [2.08-6.19] & $0.21[0.03-1.45]$ & $19.82[7.69-51.12]$ & $0.88[0.84-0.89]$ \\
\hline \multicolumn{7}{|l|}{ Mean age(y) } \\
\hline$\leq 41.6$ & $0.73[0.60-0.83]$ & $0.91[0.78-0.96]$ & 7.68 [3.47-16.99] & $0.30[0.21-3.87]$ & $25.44[13.52-47.87]$ & $0.87[0.84-0.90]$ \\
\hline$>41.6$ & $0.75[0.50-0.90]$ & $0.84[0.68-0.93]$ & $4.64[2.76-7.78]$ & $0.29[0.14-0.60]$ & $15.76[8.90-27.91]$ & $0.87[0.83-0.90]$ \\
\hline \multicolumn{7}{|l|}{ Sample size } \\
\hline$\leq 100$ & $0.81[0.59-0.93]$ & $0.77[0.64-0.86]$ & $3.47[2.46-4.88]$ & $0.25[0.12-0.54]$ & $13.88[6.71-28.73]$ & $0.84[0.81-0.87]$ \\
\hline$>100$ & $0.66[0.58-0.73]$ & $0.94[0.86-0.97]$ & $10.54[5.06-21.95]$ & $0.36[0.30-0.44]$ & 29.15 [15.14-56.15] & $0.81[0.77-0.84]$ \\
\hline \multicolumn{7}{|l|}{$\begin{array}{l}\text { Glioma } \\
\text { grading }\end{array}$} \\
\hline Low & $0.87[0.77-0.94]$ & $0.89[0.80-0.95]$ & $4.71[1.35-16.43]$ & $0.20[0.11-0.35]$ & 45.37 [14.99-137.4] & $0.93[0.91-0.96]$ \\
\hline high & $0.76[0.67-0.84]$ & $0.75[0.62-0.85]$ & 2.84 [1.36-5.93] & $0.31[0.18-0.56]$ & $10.43[4.11-26.45]$ & $0.83[0.80-0.85]$ \\
\hline
\end{tabular}

\section{DISCUSSION}

Nowadays, more fully precise excision of glioma is a challenge for neurosurgeons, the large extent of tumor resection could influence the prognosis and quality of life for patients. Ultrasound is an old and common technology, but lack of sufficient evidence to prove that is a valid method tool at characterizing glioma residual. The high sensitivity and specificity $(0.75$ and 0.88$)$ in this meta-analysis revealed that IOUS is a meritorious tool to distinguish glioma margins clearly in surgical excision, especially in low-grade glioma with larger AUC than

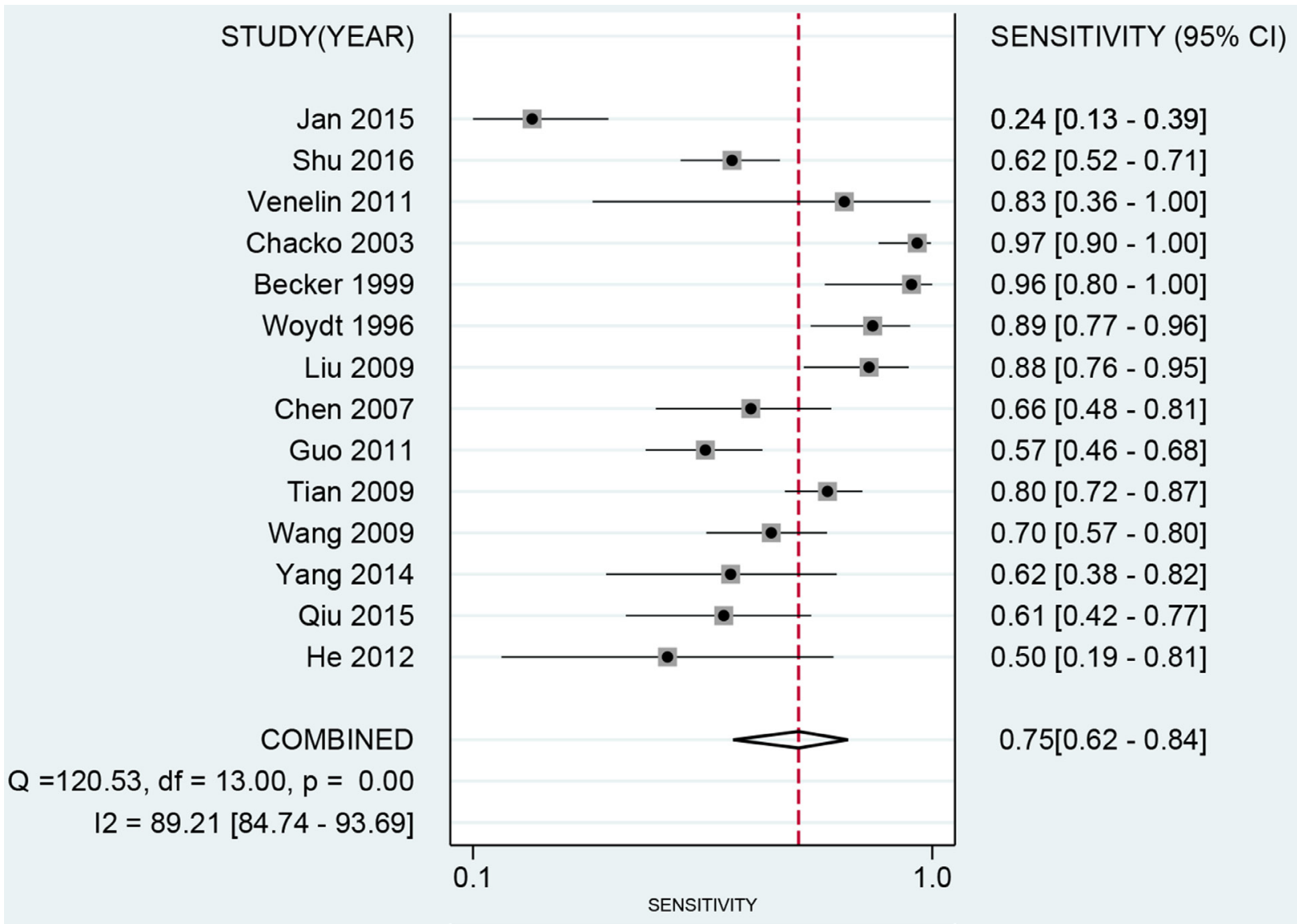

Figure 2: Forest plot of pooled sensitivity of intraoperative ultrasound for glioma residual. 


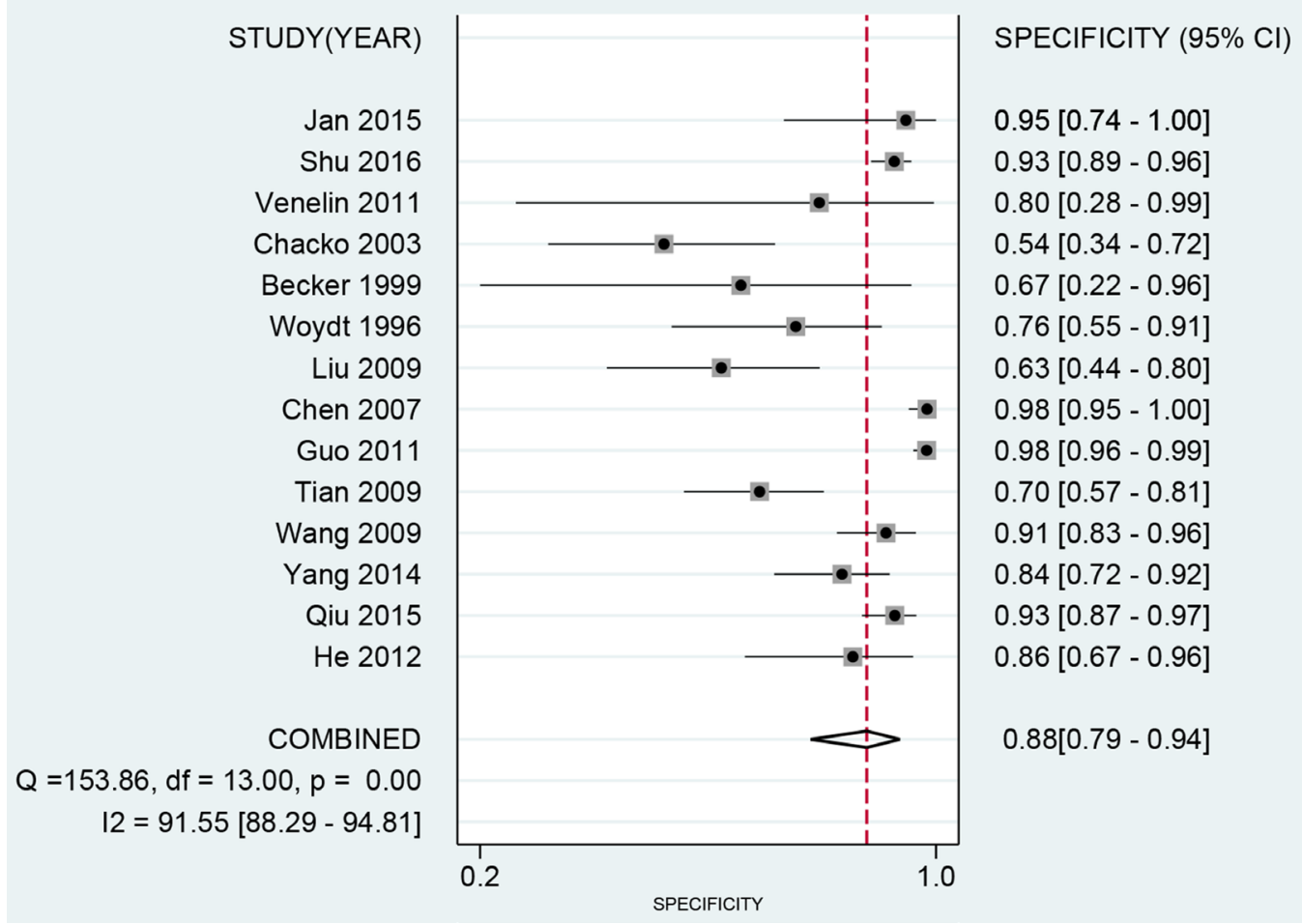

Figure 3: Forest plot of pooled specificity of intraoperative ultrasound for glioma residual.

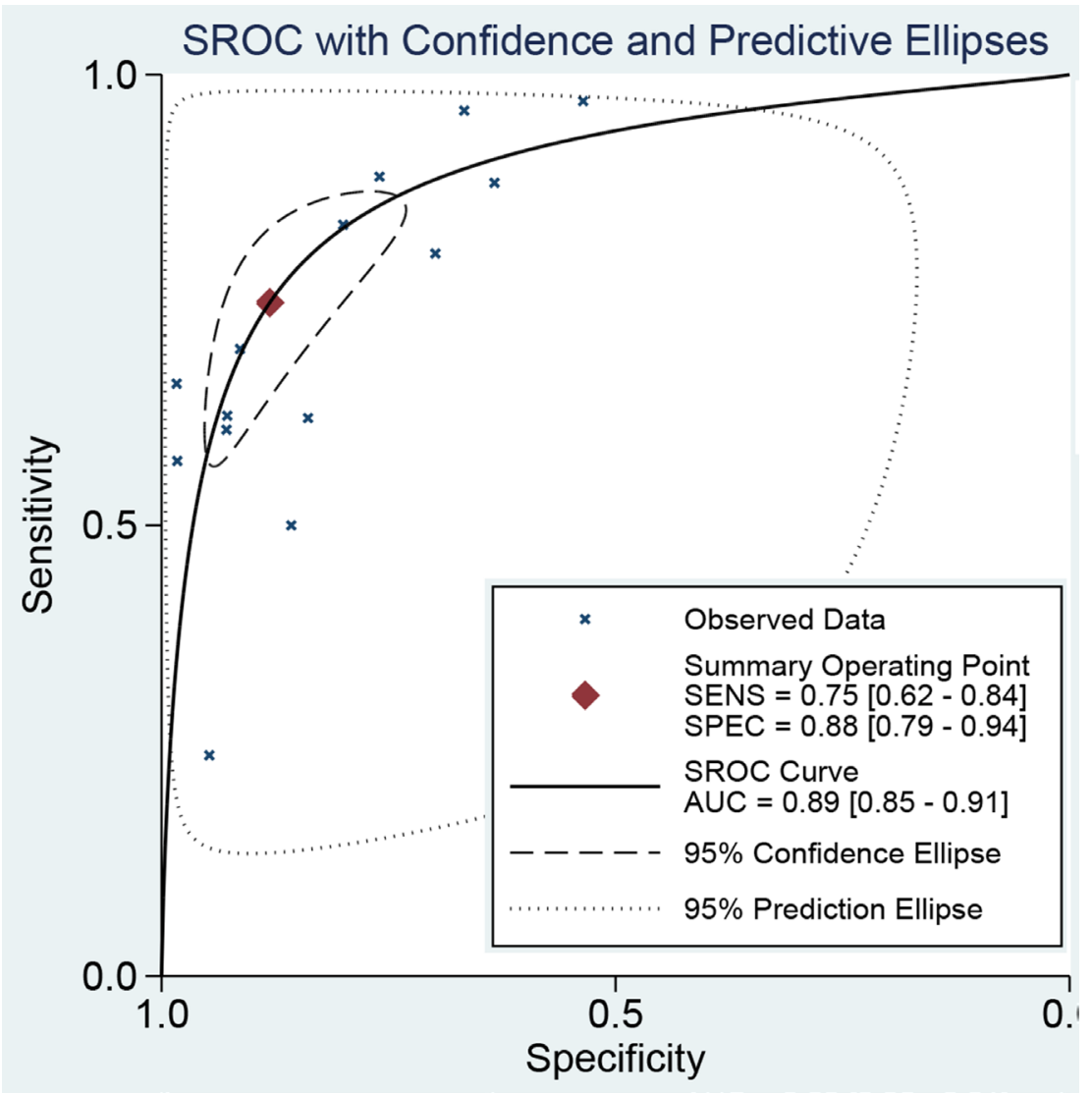

Figure 4: The SROC curve of intraoperative ultrasound for glioma residual. 
high-grade glioma. Distinguished edema from irregular shape and infiltrating growth is probably hard for highgrade glioma, even ultrasound can display tumor areas at normal blood brain barriers [24].

An appropriate diagnostic method include accuracy, real-time, low price, and effectiveness. IOUS meets these requirements and can be performed multiple times [25]. According to Woydt's tumor residual norm [10], irregular hyperecho or annular strong echo stretches into the surrounding brain tissues of residual cavity with the thickness $\geq 5 \mathrm{~mm}$ as tumor residue. We used IOUS to detect glioma location, tumor size, the distance from cortex, surrounding blood supply and para-carcinoma tissues for identifying the extent of tumor removal.
The results showed diagnostic odds ratio is 21.83 and the pooled PLRs are 6.27 and 0.29, which indicate the probability of tumor residues diagnosis compared with brain tissues is 6.27 times in case of hyperecho exceeds the cut-off value. On the contrary, the probability of definite case is $29 \%$ when it is below the cut-off value. IOUS has unique advantages, but it cannot fully replace other detection tools with the interference of ultrasonic pseudo morphism, bubbles, blood clot [26]. As we know, neuronavigation is more accurate for tumors less than 1 $\mathrm{cm}$, but the application is restricted by brain deformation or shifting after skull opening [27-28]. So an integration of IOUS into neuronavigation could offer a combine image to determine the tumor boundary.

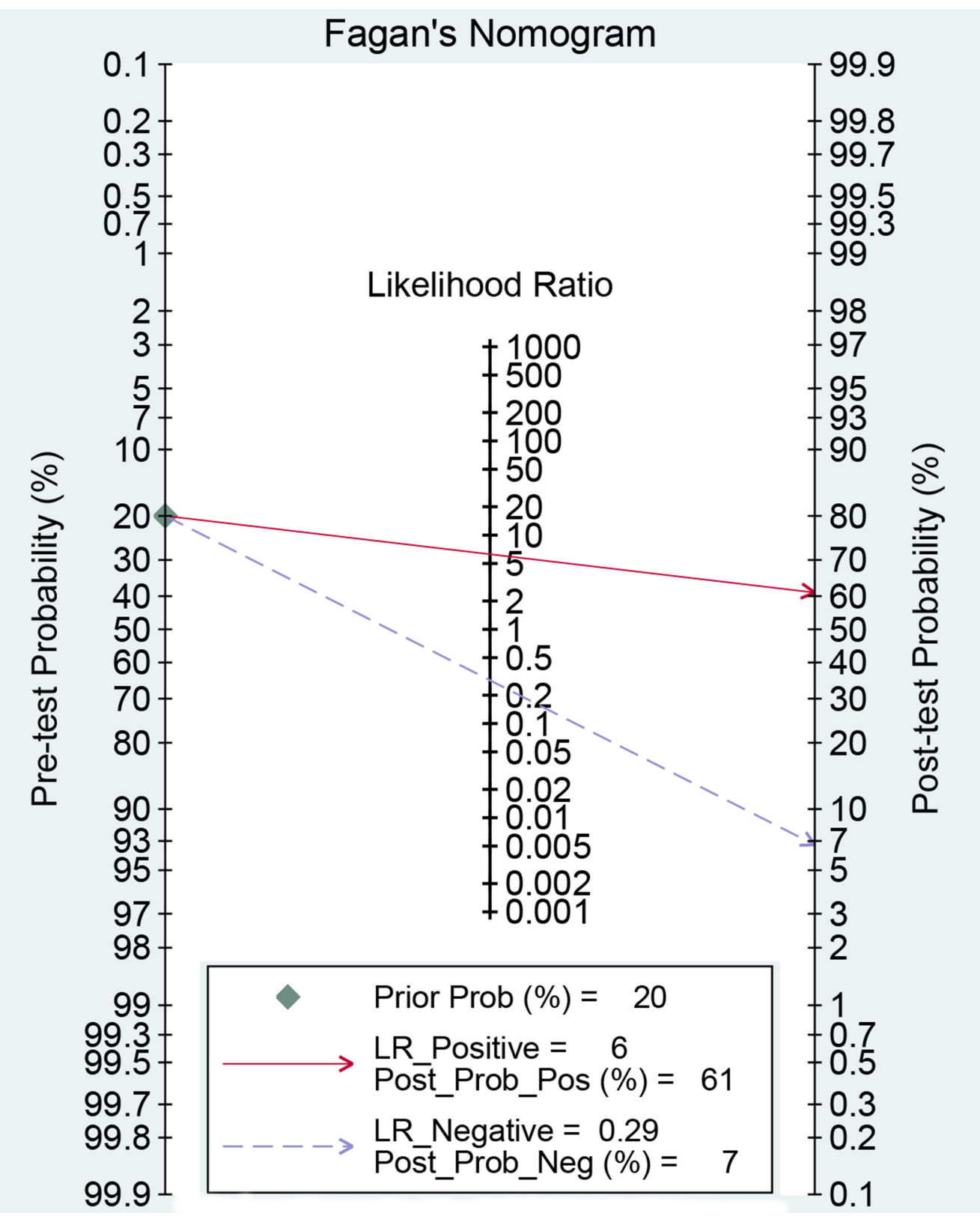

Figure 5: Fagan diagram evaluating the overall diagnostic value of intraoperative ultrasound for glioma residual. 
Now give some suggestion to avoid false positives by using proper operation and sufficient experience. (i) remove hemostasis material from the residual cavity before using ultrasound; (ii) using physiological saline to improve diagnostic accuracy; (iii) discern the echo size and position among tumor tissue, distinguish edema and colloid hyperplasia carefully; (iv) the hematoma and edema with clearer boundary presented stronger echo, but necrosis and cystic degeneration is hypoechoic. The examiner must be aware of ultrasound technology to define margins and assess the degree of resection in surgery.

In recent years, contrast enhanced ultrasound provides more anatomy and pathology information and offers clearly tumor boundary by new-type contrast agents appeared [29]. With the improvement of ultrasound technology, linear-array ultrasound has been offered to further resection in surgery operation. IOUS has become an indispensable tool to judge the degree of glioma resection, identify gliosis from gliomas and estimate tumor pathology classification [30].

In conclusion, this systematic review and metaanalysis was to evaluate the diagnostic features of intraoperative ultrasound, which has a significantly higher diagnosis accuracy. Current evidence suggests that IOUS may be a useful and non-invasive method for detecting glioma margin and allows safer removal.

\section{MATERIALS AND METHODS}

\section{Search protocol}

We conducted a literature search through following electronic databases: PubMed, Embase, Web of Science, CNKI, Wanfang and Weipu updated to May 5, 2017. Meanwhile, Google and Baidu engine were searched by comparing IOUS and pathological diagnostic criteria for treatment of glioma residues. Our research protocol consists of the detailed search strategy, screening criteria for titles, abstracts, and full-text articles. The search terms included "intraoperative ultrasound" or "ultrasonography" or "IOUS" and "intracranial glioma" or "glioma" and "residue" or "remnant". In order to find all potentially eligible articles, we searched the reference lists manually for relevant study. The Preferred Reporting Items for Systematic Reviews was used in this meta-analysis [31]. The checklist guidelines was shown in Supplementary Table 1.

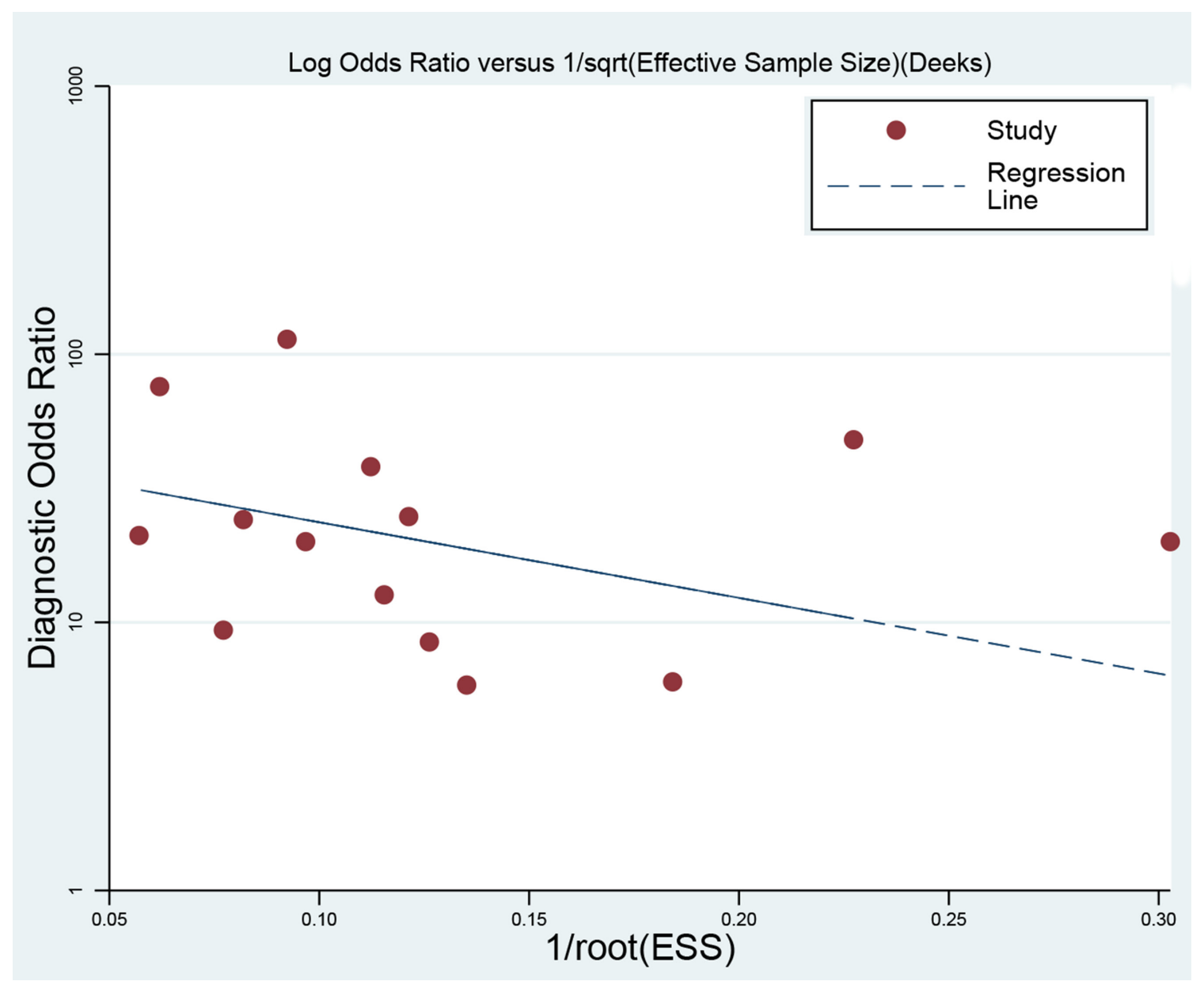

Figure 6: Deek's funnel plot to evaluate the publication bias. 


\section{Selection criteria}

The inclusion criteria were as follows: 1) all cases were performed by IOUS before harvesting pathological results; 2) the process of IOUS was evaluated; 3) raw data were sufficient to calculate true positive (TP), false positive (FP), false negative (FN) and true negative (TN); 4) abstract, review, conference paper and case report (less than 5 cases) were excluded; 5) no partial data or repetitive publication was permitted; 6) intracranial tumors were not admitted (except intracranial glioma), such as cavernous hemangioma, metastatic encephala, and radiation necrosis; 7) only human studies were included and the language was limited in English and Chinese.

\section{Data extraction and quality assessment}

According to the above criteria, two authors independently filtered the unqualified studies by reading the titles and abstracts, and then scanned full texts of the remaining articles to find the eligible one. Duplicate records were deleted. In this process, any divergence was resolved through discussion. The extracted data included article serial number, title, the name of first author, country, year of publication, mean age and sample size. Moreover, specificity, sensitivity and TP/FP/FN/TN were extracted from each eligible article or could calculate relevant data from original article.

The selected articles quality were assessed by the Quality Assessment of Diagnostic Accuracy Studies 2 (QUADAS-2) [32], which included two fields of risk of bias and applicability. Each field consists of four items: patient selection, index text, reference standard, and flow $\&$ timing. Each item contains 17 sub-clauses as questions. We scored the questions as "low", "high", or "unclear" to examine diagnostic studies.

\section{Statistical analysis}

The pooled sensitivity, specificity, diagnostic odds ratios (DOR), positive likelihood ratios (PLR), negative likelihood ratios (NLR), area under the curve (AUC) and the corresponding $95 \%$ confidence intervals (CIs) were calculated by bivariate regression model on Stata 12.0 software and Review manager 5.3. The summary receiver operator characteristic (SROC) curves were plotted for graphical assessment. Threshold effect from different diagnostic cutoff values was tested by Spearman correlation analysis. Heterogeneity was detected via $Q$ test and $\mathrm{I}^{2}$ statistic, with $\mathrm{I}^{2}>50 \%$ and $P<0.05$ indicating the presence of heterogeneity [33-34]. If heterogeneity existed, a random effects model was performed, otherwise a fixed effects model was used. And subgroup analysis was conducted to dissect the heterogeneity. Publication bias was evaluated visually by using Deek's funnel plots; the likelihood ratio, prior probability and posterior probability were tested by Fagan plot [35]. The $P<0.05$ was considered as statistical significance.

\section{Abbreviation}

IOUS, intraoperative ultrasound; TP, true positive; FP, false positive; FN, false negative; TN, true negative; QUADAS-2, Quality Assessment of Diagnostic Accuracy Studies; PLRs, positive likelihood ratios; NLRs, negative likelihood ratios; DORs, diagnostic odds ratios; SROC, summary receiver operator characteristic curve area; CI, confidence interval.

\section{ACKNOWLEDGMENTS}

ZGY and SLF designed this study and contributed substantially to the design of the search strategy. ZGY and SDL searched and selected the trials and extracted data. LZZ and ZGY performed the analysis and interpreted the data. ZGY wrote the manuscript. ZGY and SDL participated in the data extraction and critically revised it. ZGY and SLF proofread the final version.

\section{CONFLICTS OF INTEREST}

The authors declare that they have no conflict of interest.

\section{REFERENCES}

1. Ostrom QT, Gittleman H, Liao P, Rouse C, Chen Y, Dowling J, Wolinsky Y, Kruchko C, Barnholtz-Sloan J. CBTRUS statistical report: primary brain and central nervous system tumors diagnosed in the United States in 2007-2011. Neuro Oncol. 2014; 16:v1-v63.

2. Seidlitz A, Siepmann T, Löck S, Juratli T, Baumann M, Krause M. Impact of waiting time after surgery and overall time of postoperative radiochemotherapy on treatment outcome in glioblastoma multiforme.Radiation Oncology. 2015; 10:172-182.

3. Ziu M, Kalkanis SN, Gilbert M, Ryken TC, Olson JJ. The role of initial chemotherapy for the treatment of adults with diffuse low grade glioma: A systematic review and evidence-based clinical practice guideline. J Neurooncol. 2015; 125:585-607.

4. Malhi S, GU X. Nanocarrier-mediated drugs targeting cancer stem cells: an emerging delivery approach. Expert Opinion on Drug Delivery. 2015; 12:1177-1201.

5. Regelsberger J, Lohmann F, Helmke K, Westphal M. Ultrasound-guided surgery of deep seated brain lesions. Eur J Ultrasound. 2000; 12:115-121.

6. Sosna J, Barth MM, Kruskaal JB, Kane RA. Intraoperative sonography for neurosurgery. J Ultrasound Med. 2005; 24:1671-1682. 
7. Gerganov VM, Samii A, Akbarian A, Stieglitz L, Samii M, Fahlbusch R. Reliability of intraoperative high-resolution $2 \mathrm{D}$ ultrasound as an alternative to high-field strength MR imaging for tumor resection control: a prospective comparative study. J Neurosurg. 2009; 111:512-519.

8. Willems PW, Van der Sprenkel JW, Tulleken CA, Viergever MA, Taphoorn MJ. Neuronavigation and surgery of intracerebral tumours. J Neurol. 2006; 253:1123-1136.

9. Erdogan N, Tucer B, Mavili E, Menku A, Kurtsoy A. Ultrasound guidance in intracranial tumor resection: correlation with postoperative magnetic resonance findings. Acta Radiol. 2005; 46:743-749.

10. Woydt M, Krone A, Becker G, Schmidt K, Roggendorf W, Roosen K. Correlation of intra-operative ultrasound with histopathologic findings after tumor resection in supratentorial gliomas. A method to improve gross total tumor resection. Acta Neurochir. 1996; 138:1391-1398.

11. He Y, He W, Du LJ, Yu SQ, Wang JS, Li HZ. The value of intraoperative contrast-enhanced ultrasonography for improving the completely resection of cerebral gliomas. Chin J Med Ultrasound. 2012; 9:36-39.

12. Wang LS, He W, Liu HS, Lin S, Tian YJ. A comparative study for intraoperative ultrasound and pathology evaluation on resection of parenchymal brain tumors. Chin J Med Ultrasound. 2009; 6:500-506.

13. Tian YJ, Lin S, Liu HZ, Wang LS, He W, Zhang MZ, Luo L, Qin YM. Value of intraoperafive ultrasound in detecting the boundaries of Intracranial glioms. Natl Med J China. 2009; 89:1305-1308.

14. Guo SQ, Chen YH, Luo JB. The value of intraoperative ultrasound in postoperative glioma tumor residual. The Journal of Practical Medicine. 2011; 27:852-854.

15. Chen YH, Wang XY, Yu L, Ke YS, Xu RX. Application of technologically improved high resolution ultrasound in brain glioma resections. Chin J Neuro Med. 2006; 5: 702-705.

16. Liu J, Yao LH. Intraoperative Ultrasound on the evaluation of residual tumor after resection of supratentorial gliomas. Xin Jiang Med University. 2009; 8:1-37.

17. Becker G, Hofmann E, Woydt M, Hulsmann U, Maurer M, Lindner A, Becker T, Krone A. Postoperative neuroimaging of high-grade gliomas: comparison of transcranial sonography, magnetic resonance imaging, and computed tomography. Neurosurgery. 1999; 44:469-477.

18. Chacko AG, Kumar NK, Chacko G, Athyal R, Rajshekhar V. Intraoperative ultrasound in determining the extent of resection of parenchymal brain tumors - a comparative study with computed tomography and histopathology. Acta Neurochir. 2003; 145:743-748.

19. Qiu JX, Wang H. The value of contrast-enhanced ultrasound in resection of cerebral glioma. Jilin University. 2015; 4:445-461.

20. Yang GF, Mi CR, Wang W. Application value of contrastenhanced ultrasonography in judging tumor residues after brain glioma operation. J Clin Ultrasound in Med. 2014; 16:101-103.

21. Gerganov VM, Samii A, Giordano M, Samii M, Fahlbusch R. Two-dimensional high-end ultrasound imaging compared to intraoperative MRI during resection of low-grade gliomas. J Clin Neurosci. 2011; 18:669-673.

22. Yu SQ, Wang JS, Chen SY, Liu XM, Li Y, Ding YM, Li XY, Sun YL, Chen H. Diagnostic significance of intraoperative ultrasound contrast in evaluating the resection degree of brain glioma by transmission electron microscopic examination. Natl Med J China. 2015; 12:186-190.

23. Coburger J, Scheuerle A, Kapapa T, Engelke J, Thal DR, Wirtz CR, Konig R. Sensitivity and specificity of linear array intraoperative ultrasound in glioblastoma surgery: a comparative study with high field intraoperative MRI and conventional sector array ultrasound. Neurosurg Rev. 2015; 38:499-509.

24. Smith JS, Chang EF, Lamborn KR, Chang SM, Prados MD, Cha S, Tihan T, Vandenberg S, McDermott MW, Berger MS. Role of extent of resection in the long-term outcome of lowgrade hemispheric gliomas. J Clin Oncol. 2008; 26:1338-1344.

25. Miller D, Heinze S, Tirakotai W, Bozinov O, Surucu O, Benes L, Bertalanffy H, Sure U. Is the image guidance of ultrasonography beneficial for neurosurgical routine? Surg Neurol. 2007; 67:579-587.

26. Ivanov M, Wilkins S, Poeata I, Brodbelt A. Intraoperative ultra-sound in neurosurgery - a practical guide. $\mathrm{Br} \mathrm{J}$ Neurosurg. 2010; 24:510-517.

27. Fu J, Hong JD, Liu Q, Guo Y, Liao YW, Huang JH, Wen SL, Shen LF. Epithelial membrane protein 3 regulates TGF- $\beta$ signaling activation in CD44-high glioblastoma. Oncotarget. 2017; 9:14343-14358. https://doi.org/10.18632/ oncotarget.11102.

28. Roessler K, Sommer B, Grummich P, Coras R, Kasper BS, Hamer HM, Blumcke I, Stefan H, Buchfelder M. Improved resection in lesional temporal lobe epilepsy surgery using neuronavigation and intraoperative MR imaging: Favourable long term surgical and seizure outcome in 88 consecutive cases. Seizure. 2014; 23:201-207.

29. Wang YQ, Yu SQ, Wang JS, Ji N, Ren T, Li DL, Kang JL, Li HZ. Application of intraoperative contrast enhanced ultrasound in different pathological grades of glioma. Natl Med J China. 2012; 92:1495-1497.

30. Koenig RW, Schmidt TE, Heinen CP, Wirtz CR, KretschmerT, Antoniadis G, Pedro MT. Intraoperative high-resolution ultrasound: a new technique in the management of peripheral nerve disorders. J Neurosurg. 2011; 114: 514-521.

31. Moher D, Liberati A, Tetzlaff J, Altman DG. PRISMA Group: Preferred Reporting Items for Systematic Reviews and Meta-Analyses: The PRISMA Statement. PLoS Med. 2009; 6:e1000097.

32. Whiting PF, Rutjes AW, Westwood ME, Mallett S, Deeks JJ, Reitsma JB, Leeflang MM, Sterne JA, Bossuyt PM. 
QUADAS-2: a revised tool for the quality assessment of diagnostic accuracy studies. Ann Intern Med. 2011; 155:529-536.

33. Reitsma JB, Glas AS, Rutjes AW, Scholten RJ, Bossuyt PM, Zwinderman AH. Bivariate analysis of sensitivity and specificity produces informative summary measures in diagnostic reviews. J Clin Epidemiol. 2005; 58:982-990.

34. Li ZZ, Zhou Q, Li YY, Yan SP, Fu J, Huang XQ, Shen LF. Mean cerebral blood volume is an effective diagnostic index of recurrent and radiation injury in glioma patients: A meta-analysis of diagnostic test. Oncotarget. 2017; 8:15642-15650. https://doi.org/10.18632/oncotarget.14922.

35. Egger M, Davey SG, Schneider M, Minder C. Bias in metaanalysis detected by a simple, graphical test. BMJ. 1997; 315:629-634. 